\title{
GEMS: The destiny of Blue Spheroidal Galaxies
}

\author{
Boris Häußler ${ }^{1}$, Eric F. Bell ${ }^{1}$, Marco Barden ${ }^{1}$, Daniel H. McIntosh ${ }^{2}$, \\ Hans-Walter Rix ${ }^{1}$, Andrea Borch ${ }^{3}$, Steven V. W. Beckwith ${ }^{4,5}$, \\ John A. R. Caldwell ${ }^{6}$, Catherine Heymans ${ }^{7}$, Knud Jahnke ${ }^{1}$, \\ Shardha Jogee ${ }^{8}$, Sergey E. Koposov ${ }^{1}$, Klaus Meisenheimer ${ }^{1}$, \\ Chien Y. Peng ${ }^{4}$, Sebastian F. Sánchez ${ }^{9}$, Rachel S. Somerville ${ }^{1}$, \\ Lutz Wisotzki ${ }^{10}$, and Christian Wolf ${ }^{11}$ \\ ${ }^{1}$ Max-Planck-Institut für Astronomie, Königstuhl 17, 69117, Heidelberg, Germany \\ ${ }^{2}$ Department of Astronomy, University of Massachusetts, 710 North Pleasant Street, Amherst, \\ MA 01003, USA \\ ${ }^{3}$ Astronomisches Recheninstitut, Mönchhofstraße 12-14, 69120, Heidelberg, Germany \\ ${ }^{4}$ Space Telescope Science Institute, 3700 San Martin Dr., Baltimore, MD 21218, USA \\ ${ }^{5}$ Johns Hopkins University, 3400 North Charles Street, Baltimore, MD 21218, USA \\ ${ }^{6}$ University of Texas, McDonald Observatory, Fort Davis, TX 79734, USA \\ ${ }^{7}$ Department of Physics and Astronomy, The University of British Columbia, 6224 \\ Agricultural Road Vancouver, V6T 1Z1 Canada \\ ${ }^{8}$ University of Texas at Austin, 1, University Station C1400, Austin, TX 78712-0259, USA \\ ${ }^{9}$ Centro Astronomico Hispano Aleman de Calar Alto, C/Jesus Durban Remon 2-2, Almeria, \\ E-04004, Spain \\ ${ }^{10}$ Universität Potsdam, Am Neuen Palais 10, 14469, Potsdam, Germany \\ ${ }^{11}$ Department of Physics, Denys Wilkinson Bldg., University of Oxford, Keble Road, Oxford, \\ OX1 3RH, UK \\ email: boris@mpia.de
}

One of the key predictions of hierarchical galaxy formation models is that a significant fraction of elliptical galaxies form in late merging events. One of the most important diagnostics of such an assembly is the existence of blue spheroidal galaxies, which have spheroid-dominated morphologies and blue colors indicating recent star formation, as an intermediate step in the evolution of elliptical galaxies.

We present results from the GEMS (Rix et al. 2004) survey showing the properties of these galaxies derived from 2-D galaxy fitting using GALFIT (Peng et al. 2002) of the $\sim 8000$ galaxies with photometric redshifts in the $28 \times 28$ HST mosaic. For the first time we were able to divide the observed population of blue spheroidal galaxies into subpopulations of different stellar masses.

We find that massive blue spheroidals are likely to be the progenitors of red sequence galaxies. In contrast, low-mass blue spheroidals have half-light radii considerably in excess of those measured for low-mass present day elliptical galaxies; instead, they have larger sizes similar to present-day disk-dominated systems with substantial bulges.

\section{References}

Peng, C.Y., Ho, L.C., Impey, C.D., \& Rix, H., 2002, AJ, 124:266-293.

Rix, H., Barden, M., Beckwith, S.V.W., Bell, E.F., Caldwell, J.A.R., Häußler, B., Jahnke, K., Jogee, S., McIntosh, D.H., Meisenheimer, K., Peng, C.Y., Sanchez, S.F., Somerville, R.S., Wisotzki, L., \& Wolf, C., 2004, ApJS, 152:163. 